\title{
The Number of Lines Tangent to Arbitrary Convex Polyhedra in 3D
}

\author{
H. Brönnimann \\ O. Devillers" \\ S. Lazard\$ \\ H.-S. Na**
}

\author{
H. Everett ${ }^{\S} \quad$ M. Glisse ${ }^{\S}$ \\ S. Whitesides $\ddagger$
}

X. Goaoc ${ }^{\S}$

\begin{abstract}
We prove that the lines tangent to four possibly intersecting convex polyhedra in $\mathbb{R}^{3}$ with $n$ edges in total form $\Theta\left(n^{2}\right)$ connected components in the worst case. In the generic case, each connected component is a single line, but our result still holds for arbitrary degenerate scenes. More generally, we show that a set of $k$ convex polyhedra with a total of $n$ edges admits, in the worst case, $\Theta\left(n^{2} k^{2}\right)$ connected components of (possibly occluded) lines tangent to any four of these polyhedra. We also show a lower bound of $\Omega\left(n^{2} k^{2}\right)$ on the number of non-occluded maximal line segments tangent to any four of these $k$ convex polyhedra.
\end{abstract}

\section{Categories and Subject Descriptors}

F.2.2 [Nonnumerical Algorithms and Problems]: Geometrical problems and computations; G.2.1 [Combinatorics] Counting problems; I.3.5 [Computer Graphics]: Computational Geometry and Object Modeling; I.3.7 [ThreeDimensional Graphics and Realism]: Visible line/surface algorithms

\section{General Terms}

Algorithms, Theory.

\section{Keywords}

Computational geometry, 3D visibility, visual events, visibility complex.

\section{INTRODUCTION}

Computing visibility relations in a $3 \mathrm{D}$ environment is a central problem of computer graphics (e.g. determining the view from a given point, identifying the set of blockers between two polygons, computing the umbra and penumbra

\footnotetext{
${ }^{\dagger}$ Polytechnic University, hbr@poly.edu

${ }^{\ddagger}$ McGill University, \{vida,sue\}@cs.mcgill.ca

${ }^{\S}$ LORIA - INRIA Lorraine, \{name\}@loria.fr.

"INRIA Sophia-Antipolis, Olivier.Devillers@inria.fr.

**Soongsil University, hsnaa@computing.ssu.ac.kr
}

Permission to make digital or hard copies of all or part of this work for personal or classroom use is granted without fee provided that copies are not made or distributed for profit or commercial advantage and that copies bear this notice and the full citation on the first page. To copy otherwise, to republish, to post on servers or to redistribute to lists, requires prior specific permission and/or a fee.

SCG'04, June 8-11, 2004, Brooklyn, New York, USA.

Copyright 2004 ACM 1-58113-885-7/04/0006 ...\$5.00.

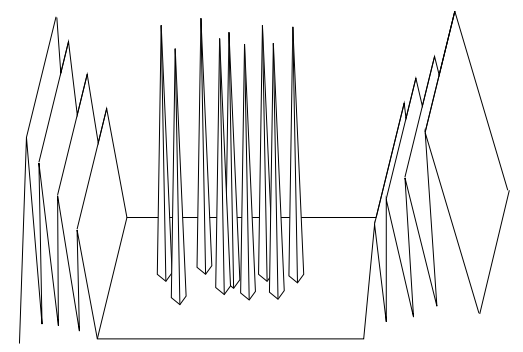

Figure 1: A terrain of size $O(n)$ with $\Omega\left(n^{4}\right)$ maximal non-occluded line segments tangent in four points.

cast by an area light source) and other engineering tasks (e.g. radio propagation simulation). Visibility is well-known to account for a significant portion of the cost of the overall computation in these applications, and consequently a large body of research is devoted to speeding up visibility computations through the use of data structures (see [7] for a survey, including space partitions, aspect graphs, and visual hulls).

One such structure, the visibility complex [11], which has been proposed for rendering [9], encodes visibility relations by partitioning the set of maximal free line segments. Unfortunately the complex is potentially enormous which has so far prevented its application in practice. Its size is intimately related to the number of lines tangent to four objects in the scene; for $n$ triangles in $\mathbb{R}^{3}$, the complex can have size $\Theta\left(n^{4}\right)$ in the worst case [6], even when the triangles form a terrain (see Figure 1).

There is evidence, both theoretical [5] and practical [8], that this bound is largely pessimistic in practical situations. The lower bound examples are carefully manufactured to exhibit the worst-case behavior, and often require an unrealistically large ratio between longest and shortest edges. On the other hand, for random scenes, Devillers et al. [5] prove that the expected size of the visibility complex is much smaller; for uniformly distributed unit balls the expected size is linear and for polygons or polyhedra of bounded aspect ratio it is at most quadratic.

While these results are encouraging, most scenes are not random. In fact, most scenes have a lot of structure which we can exploit; a scene is typically represented by many triangles which form a much smaller number of convex patches. If a scene consists of $k$ disjoint convex polyhedra with $n$ edges in total then, under a strong general position assumption, the number of tangents to four of the polyhedra is at most $O\left(n^{2} k^{2}\right)$; this follows directly from the bound proved in [10] on the number of combinatorial changes of the sil- 
houette map viewed from a point moving along a straight line, and was also later proved in [3].

Our results. In this paper, we generalize the result of [3, 10] in two ways. First, we consider polytopes (i.e., convex polyhedra) that may intersect. We show that among $k$ polytopes of total complexity $n$, the number of lines tangent to any four of them is in the worst case either infinite or $\Theta\left(n^{2} k^{2}\right)$. The most surprising aspect of this result is that the bound (which is tight) is the same whether the polytopes intersect or not [3]. This is in sharp contrast to the 2D case, where the number of tangents of two convex polygons is always 4 if disjoint, and could be linear in the size of the polygons if they intersect.

Secondly we consider polytopes in arbitrary position: we drop all general position assumptions. Four such objects may admit an infinite number of common tangents which can be partitioned in line space into connected components. Two lines are said to be in the same connected component if and only if their corresponding points in line space (e.g., Plücker space) are in the same connected component of the set of points corresponding to all the lines tangent to any 4 among the $k$ objects; equivalently one line can be continuously moved into the other while remaining tangent to at least four objects ${ }^{1}$.

Our main results are the following.

THEOREM 1. Given 4 polytopes in $\mathbb{R}^{3}$ with $n$ edges in total, the number of lines tangent to the 4 polytopes is, in the worst case, either infinite or $\Theta\left(n^{2}\right)$. More generally, these lines form $\Theta\left(n^{2}\right)$ connected components. Moreover, if one of the polytopes has $m$ edges, these bounds improve to $\Theta(m n)$.

This result improves the previously known upper bound of $O\left(n^{3} \log n\right)$ which follows from the same bound on the complexity of the set of line transversals to a set of polyhedra (here four) of total complexity $O(n)[1]$

TheOREM 2. Given $k$ polytopes in $\mathbb{R}^{3}$ with $n$ edges in total, the lines (possibly occluded) tangent to any four of the polytopes form, in the worst case, $\Theta\left(n^{2} k^{2}\right)$ connected components.

This result improves the trivial bound of $O\left(n^{4}\right)$.

Our results hold for any set of polytopes including those that degenerate to a polygon, segment or point ${ }^{2}$. The polytopes may intersect in any way; they may overlap or coincide and their faces may be triangulated.

To emphasize the importance of considering intersecting polytopes, observe that computer graphics scenes usually consist of not necessarily convex objects which can, however, be decomposed into sets of convex polyhedra. Notice that simply decomposing these objects into convex polyhedra with disjoint interiors may induce a scene of much higher complexity than a decomposition into intersecting polytopes. Moreover, the decomposition of a polyhedron into interior-disjoint polytopes may yield new tangents which

\footnotetext{
${ }^{1}$ Notice that the set of objects the line is tangent to might change during the motion.

${ }^{2} \mathrm{~A}$ line is tangent to a polytope with non-empty interior if and only if it intersects the polytope but not its interior. We also use this definition if the polytope is a segment or a point; if the polytope is a polygon then a line is a tangent line if and only if it intersects the $2 \mathrm{D}$ boundary of the polygon.
}

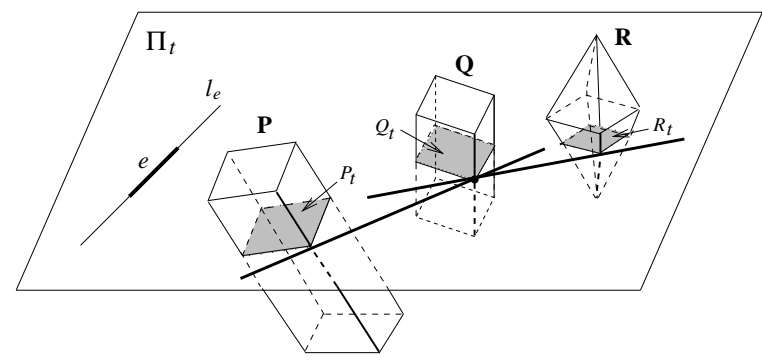

Figure 2: Plane $\Pi_{t}$ contains edge $e$ and intersects polytopes $\mathbf{P}, \mathbf{Q}$, and $\mathbf{R}$ in polygons $P_{t}, Q_{t}$, and $R_{t}$, respectively.

were not present in the original scene; indeed a line tangent to two polytopes along a shared face is not tangent to their union.

The importance of considering polytopes in arbitrary position comes from the fact that graphics scenes are full of degeneracies both in the sense that four polytopes may admit infinitely many tangents and that polytopes may share edges or faces. Note that we could not find a perturbation argument that guarantees the preservation of all connected components of tangents and we do not believe it is a simple matter.

The paper is organized as follows. We prove the upper bounds of Theorems 1 and 2 in Sections 2 and 3, and the lower bounds in Section 4.

\section{MAIN LEMMA}

We prove in this section the following lemma which yields the upper bounds in Theorem 1. This lemma is also fundamental for the proof of the upper bound of Theorem 2, which we provide in Section 3.

Consider three polytopes $\mathbf{P}, \mathbf{Q}$, and $\mathbf{R}$ in $\mathbb{R}^{3}$, with $p, q$, and $r$ edges, respectively, and let $e$ be an edge of a fourth polytope $\mathbf{S}$.

Main Lemma. The lines intersecting e and tangent to $\mathbf{P}$, $\mathbf{Q}, \mathbf{R}$ and $\mathbf{S}$ form $O(p+q+r)$ connected components.

The upper bounds of Theorem 1 follow from the Main Lemma. Indeed, if $s$ denotes the number of edges of $\mathbf{S}$, by summing over all these edges, the number of connected components of lines tangent to the four polytopes is $O(s) p+$ $q+r))=O\left(n^{2}\right)$. Moreover, if one of the polytopes has $m$ edges, choosing $\mathbf{S}$ to be this polytope yields that the lines tangent to the four polytopes form $O(m n)$ connected components.

The actual proof of the Main Lemma is rather complicated because it handles polytopes which may intersect in any way. In addition, the proof handles all the degenerate cases. To assist the reader, we first give an overview of the proof.

\subsection{Proof overview}

The proof is inspired by a method which was, to our knowledge, first used in [2] (see also Schiffenbauer's survey [12]).

We sweep the space with a plane $\Pi_{t}$ rotating about the line containing $e$. The sweep plane intersects the three polytopes $\mathbf{P}, \mathbf{Q}$, and $\mathbf{R}$ in three, possibly degenerate or empty, convex polygons denoted $P_{t}, Q_{t}$, and $R_{t}$, respectively (see Figure 2).

During the sweep, we track the bitangents, that are the lines tangent to $P_{t}$ and $Q_{t}$, or to $Q_{t}$ and $R_{t}$. As the sweep 

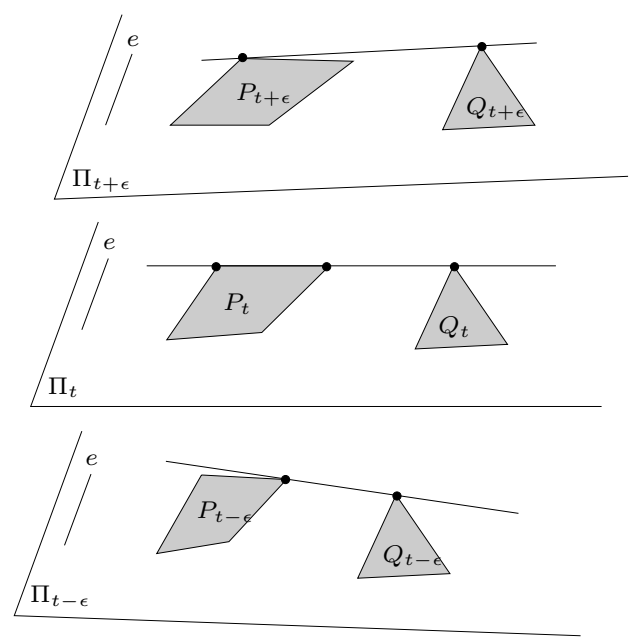

Figure 3: A bitangent to $P_{t}$ and $Q_{t}$ is tangent to $P_{t}$ along an edge. The plane $\Pi_{t}$ is F-critical.

plane rotates, the three polygons deform and the bitangents move accordingly. Every time two bitangents become aligned during the sweep, their supporting line is tangent to $\mathbf{P}, \mathbf{Q}$, and $\mathbf{R}$. A bitangent to two polygons, say $P_{t}$ and $Q_{t}$, goes through a set of vertices of these polygons. The edges of $\mathbf{P}$ and $\mathbf{Q}$ that contain these vertices are called support edges of the bitangent. (For the purpose of this overview we ignore the case where bitangents go through vertices of $\mathbf{P}$ or $\mathbf{Q})$.

In any given instance of the sweep plane $\Pi_{t}$, we consider the pairs of bitangents (one involving $P_{t}$ and $Q_{t}$, and the other $Q_{t}$ and $R_{t}$ ) that share a vertex of $Q_{t}$ (see Figure 2). The sets of edges that consist of $e$ and the support edges of two such bitangents are called candidate tuples. A candidate tuple contains at least 4 edges, $e$ and at least one edge from each polytope; most of the time, it contains exactly 4 edges.

A candidate tuple induced by an instance of the sweep plane changes as the plane rotates only when a support edge of a bitangent changes. We define critical planes in such a way that the support edges of the bitangents do not change as the sweep plane rotates in between two consecutive critical planes. As the sweep plane rotates, the support edges of a bitangent change if a support edge starts or ceases to be swept, or if, during its motion, the bitangent becomes tangent to one of the polygons along an edge (see Figure 3 ). We thus define two types of critical planes. An instance of the sweep plane is called $V$-critical if it contains a vertex of one of the polytopes, and F-critical if it contains a line that lies in the plane containing a face of one of the polytopes and is tangent to another of the polytopes (see Figures 3 and 4$)$. We will show that the number of critical planes is $O(p+q+r)$.

Notice that when the polytopes intersect there may exist a linear number of bitangents in an instance of the sweep plane; two intersecting convex polygons may admit a linear number of bitangents. Thus there can be a linear number of candidate tuples induced by any instance of the sweep plane and the linear number of critical planes leads to a quadratic bound on the total number of distinct candidate tuples. In the detailed proof of the lemma (Section 2.2), we amortize the count of candidate tuples over all the critical planes to get a linear bound on the number of distinct candidate tuples and thus on the number of connected components of lines intersecting $e$ and tangent to $\mathbf{P}, \mathbf{Q}$, and $\mathbf{R}$.

\subsection{Proof of the Main Lemma}

We now give a detailed proof of the Main Lemma for the general case of possibly intersecting polytopes in any configuration.

Since any line tangent to a polytope will remain tangent whether or not the faces are triangulated (or otherwise subdivided), we assume without loss of generality that no two faces of $\mathbf{P}$ are coplanar, and similarly for $\mathbf{Q}, \mathbf{R}$ and $\mathbf{S}$.

We can also assume without loss of generality that $\mathbf{P}, \mathbf{Q}$, $\mathbf{R}$ and $\mathbf{S}$ have non-empty interior. Indeed, if some polytopes have empty interior, they can be "inflated" as follows so that there are not more connected components of lines tangent to the four polytopes before the inflation than after ${ }^{3}$. If, say, $\mathbf{P}$ is a segment (resp. a point) it can be first transformed into any triangle having this segment (resp. point) as an edge (resp. vertex) without losing any common tangent line to the four polytopes. Now, if $\mathbf{P}$ is a polygon it can be "inflated" by adding a vertex that projects orthogonally onto $\mathbf{P}$. Let $\mathbf{P}^{\prime}$ and $\mathbf{P}^{\prime \prime}$ be two inflated instances of $\mathbf{P}$ for which the added vertices are added on opposite sides of $\mathbf{P}$ respectively. Then any line tangent to $\mathbf{P}$ is tangent to $\mathbf{P}^{\prime}$ or $\mathbf{P}^{\prime \prime}$

Let $l_{e}$ be the line containing $e$ and let $\Pi_{t}$ denote the sweep plane parameterized by $t \in[0, \pi]$ such that $\Pi_{t}$ contains the line $l_{e}$ for all $t$ and $\Pi_{0}=\Pi_{\pi}$. Each plane $\Pi_{t}$ intersects the three polytopes $\mathbf{P}, \mathbf{Q}$, and $\mathbf{R}$ in three, possibly degenerate or empty, convex polygons, $P_{t}, Q_{t}$, and $R_{t}$, respectively (see Figure 2).

A line intersecting $e$ and tangent to $\mathbf{P}, \mathbf{Q}, \mathbf{R}$ and $\mathbf{S}$ is called a generic tangent line if it is tangent to $P_{t}, Q_{t}$, and $R_{t}$ in some plane $\Pi_{t}$. Otherwise it is called a nongeneric tangent line. A nongeneric tangent line properly ${ }^{4}$ intersects $P_{t}, Q_{t}$, or $R_{t}$ in some plane $\Pi_{t}$. In this case $P_{t}, Q_{t}$, or $R_{t}$ is a face or an edge of $\mathbf{P}, \mathbf{Q}$, or $\mathbf{R}$ lying in $\Pi_{t}$, a degenerate situation.

We bound in the two following subsections the number of generic and non-generic tangent lines.

\section{Generic tangent lines}

We start with some definitions. We call support vertex of a line, a vertex of $\mathbf{P}, \mathbf{Q}$, or $\mathbf{R}$ that lies on the line. We call support edge of a line, an edge of $\mathbf{P}, \mathbf{Q}$, or $\mathbf{R}$ that intersects the line and has no endpoint on it. A support is a support edge or support vertex.

For any $t$, a bitangent to polygons $P_{t}$ and $Q_{t}$ is a line tangent to $P_{t}$ and $Q_{t}$ in $\Pi_{t}$ (the line may intersect the polygon $R_{t}$ in any way). For any $t$, let a $\left(P_{t}, Q_{t}\right)$-tuple be the nonordered set of all supports in $\mathbf{P}$ and $\mathbf{Q}$ of one of the bitangents to polygons $P_{t}$ and $Q_{t}$. Note that a $\left(P_{t}, Q_{t}\right)$-tuple consists of exactly one support in $\mathbf{P}$ and one support in $\mathbf{Q}$ except when the corresponding bitangent is tangent to $\mathbf{P}$ (or $\mathbf{Q}$ ) along a face; then the $\left(P_{t}, Q_{t}\right)$-tuple contains two supports in $\mathbf{P}$ (or $\mathbf{Q}$ ) instead of one. A $\mathbf{P Q}$-tuple is a set of edges and vertices that is a $\left(P_{t}, Q_{t}\right)$-tuple for some $t$. We define similarly the $\left(Q_{t}, R_{t}\right)$-tuples and $\mathbf{Q R}$-tuples.

\footnotetext{
${ }^{3}$ This simple argument actually only holds for extremal tangent lines ; for the general argument, see the full paper.

${ }^{4} \mathrm{~A}$ line properly intersects a polygon if and only if it intersects its relative interior.
} 


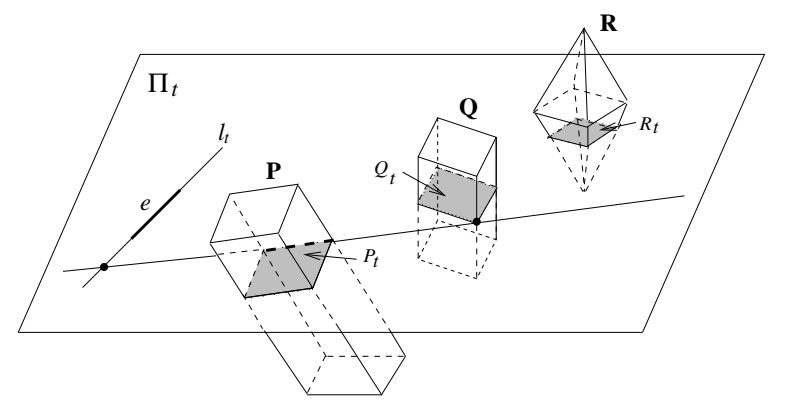

Figure 4: Plane $\Pi_{t}$ contains a line that is tangent to $\mathrm{Q}$ and lies in a plane containing a face of $\mathrm{P}$. Plane $\Pi_{t}$ is F-critical.

We say that a $\left(P_{t}, Q_{t}\right)$-tuple is maximal for some $t$ if it is not contained in any other $\left(P_{t}, Q_{t}\right)$-tuple, for the same $t$. Note that a $\left(P_{t}, Q_{t}\right)$-tuple is non-maximal for some $t$ only if all its supports intersects $\Pi_{t}$ in one and the same point (see Figure 6(b)).

For any $t$, let a $\left(P_{t}, Q_{t}, R_{t}\right)$-tuple be the union of a $\left(P_{t}, Q_{t}\right)$ tuple and a $\left(Q_{t}, R_{t}\right)$-tuple that share at least a support in Q. A $\left(P_{t}, Q_{t}, R_{t}\right)$-tuple is maximal for some $t$ if it is not contained in any other $\left(P_{t}, Q_{t}, R_{t}\right)$-tuple, for the same $t$. A PQR-tuple is a set of edges and vertices that is a $\left(P_{t}, Q_{t}, R_{t}\right)$-tuple for some $t$. Note that a PQR-tuple typically consists of three supports, one from each polytope, and consists, in all cases, of at most two supports in $\mathbf{P}$, at most three supports in $\mathbf{Q}$, and at most two supports in $\mathbf{R}$.

LEMMA 3. The set of supports of a generic tangent line is a PQR-tuple.

Proof. Any generic tangent line $\ell$ is tangent in $\Pi_{t}$ to $P_{t}$, $Q_{t}$, and $R_{t}$ for some value $t$. Thus the set of supports of $\ell$ in $\mathbf{P}$ and $\mathbf{Q}$ (resp. in $\mathbf{Q}$ and $\mathbf{R}$ ) is a $\left(P_{t}, Q_{t}\right)$-tuple (resp. a $\left(Q_{t}, R_{t}\right)$-tuple). Moreover these $\left(P_{t}, Q_{t}\right)$-tuple and $\left(Q_{t}, R_{t}\right)$ tuple contain the same supports in $\mathbf{Q}$, thus their union is a $\left(P_{t}, Q_{t}, R_{t}\right)$-tuple, hence a PQR-tuple.

We now define the critical planes $\Pi_{t}$ in such a way that the set of $\left(P_{t}, Q_{t}, R_{t}\right)$-tuples is invariant for $t$ ranging strictly in between two consecutive critical values. We introduce two types of critical planes: the $V$-critical and $F$-critical planes.

A plane $\Pi_{t}$ is $V$-critical if it contains a vertex of $\mathbf{P}, \mathbf{Q}$, or $\mathbf{R}$, not ${ }^{5}$ on $l_{e}$. A plane $\Pi_{t}$ is $F$-critical relative to polytopes $(\mathbf{P}, \mathbf{Q})$ if (see Figure 4$)$ it contains a line $\ell$ such that

(i) $\ell$ lies $^{6}$ in a plane $\Psi \neq \Pi_{t}$ containing a face of $\mathbf{P}$, and

(ii) $\ell$ is tangent in $\Psi$ to polygon ${ }^{7} \mathbf{Q} \cap \Psi$ or $\mathbf{P} \cap \Psi$, at some point not on $l_{e}$.

\footnotetext{
${ }^{5}$ This constraint ensures that the number of $\mathrm{V}$-critical planes is finite even in degenerate configurations.

${ }^{6}$ For simplicity, we do not require that $\ell$ is tangent to $\mathbf{P}$; this leads to overestimating the number of common tangents to $\mathbf{P}, \mathbf{Q}, \mathbf{R}$, and $\mathbf{S}$ but only by an asymptotically negligible amount.

${ }^{7}$ Note that not all lines in $\Psi$ tangent to $\mathbf{Q}$ are tangent to the polygon $\mathbf{Q} \cap \Psi$ when that polygon is a face or edge of Q lying in $\Psi$. Note also that we define $\Pi_{t}$ to be F-critical when $\ell$ is tangent to $\mathbf{P} \cap \Psi$ at some point not on $l_{e}$ only for handling the very degenerate case where $\mathbf{Q} \cap \Psi$ is an edge of $\mathbf{Q}$, and there exists a line in $\Psi$ that properly intersects $\mathbf{Q} \cap \Psi$ and is tangent to $\mathbf{P} \cap \Psi$ along an edge that has an endpoint on $l_{e}$ (see Figure 10 at the end of the paper).
}

F-critical planes relative to $(\mathbf{Q}, \mathbf{P}),(\mathbf{Q}, \mathbf{R})$, and $(\mathbf{R}, \mathbf{Q})$ are defined similarly. A plane $\Pi_{t}$ is F-critical if it is F-critical relative to polytopes $(\mathbf{P}, \mathbf{Q}),(\mathbf{Q}, \mathbf{P}),(\mathbf{Q}, \mathbf{R})$, or $(\mathbf{R}, \mathbf{Q})$.

The values of $t$ corresponding to critical planes $\Pi_{t}$ are called critical values. We call $V$-critical and $F$-critical events the couples $(t, o)$ where $t$ is a critical value and $o$ is a vertex or line depending on the type of critical event. In a $\mathrm{V}$-critical event, $o$ is a vertex of $\mathbf{P}, \mathbf{Q}$, or $\mathbf{R}$ that belongs to $\Pi_{t} \backslash l_{e}$. In an F-critical event, $o$ is a line lying in some plane $\Pi_{t}$ and satisfying Conditions (i-ii) above. A critical event is a V-critical or F-critical event.

Lemma 4. There are at most $\frac{2}{3}(p+q+r) V$-critical events and $\frac{8}{3}(p+2 q+r)$ F-critical events.

Proof. The number of $\mathrm{V}$-critical events is at most the total number of vertices of $\mathbf{P}, \mathbf{Q}$, and $\mathbf{R}$, and hence is less than two thirds the total number of edges of $\mathbf{P}, \mathbf{Q}$, and $\mathbf{R}$.

We now count the number of $\mathrm{F}$-critical events relative to polytopes $(\mathbf{P}, \mathbf{Q})$. Let $\Psi$ be a plane containing a face of $\mathbf{P}$. Let $\ell$ be a line lying in some plane $\Pi_{t}$ and satisfying with plane $\Psi$ Conditions (i-ii). Plane $\Psi$ does not contain $l_{e}$ because otherwise both $l_{e}$ and $\ell$ lie in the two distinct planes $\Psi$ and $\Pi_{t}$, so $\ell=l_{e}$ but then $\ell$ cannot satisfy Condition (ii). Furthermore $\ell$ and $l_{e}$ intersect or are parallel since they both lie in $\Pi_{t}$. Thus if $\Psi \cap l_{e}$ is a point then $\ell$ contains it, and otherwise $\Psi \cap l_{e}=\emptyset$ and $\ell$ is parallel to $l_{e}$.

There are at most four lines $\ell$ in plane $\Psi$ going through $\Psi \cap l_{e}$ if this intersection is a point and parallel to $l_{e}$ otherwise, and tangent to $\mathbf{Q} \cap \Psi$ or $\mathbf{P} \cap \Psi$, at some point not on $l_{e}$. Moreover each such line is contained in a unique plane $\Pi_{t}$, for $t \in[0, \pi]$, since $\ell \neq l_{e}(\ell$ contains a point not on $\left.l_{e}\right)$. Hence, a face of $\mathbf{P}$ generates at most four $\mathrm{F}$-critical events relative to $(\mathbf{P}, \mathbf{Q})$. Therefore the number of critical events relative to $(\mathbf{P}, \mathbf{Q})$ is at most $\frac{8}{3} p$ since the number of faces of a polytope is at most two thirds the number of its edges. Hence the number of critical events relative to $(\mathbf{P}, \mathbf{Q})$, $(\mathbf{Q}, \mathbf{P}),(\mathbf{Q}, \mathbf{R})$ and $(\mathbf{R}, \mathbf{Q})$ is at most $\frac{8}{3}(p+2 q+r)$.

We now prove that the critical planes have the desired property. Let $u_{e}$ be the set of supports of $l_{e}$ in $\mathbf{P}$ and $\mathbf{Q}$.

Lemma 5. If $u \neq u_{e}$ is a maximal ${ }^{8}\left(P_{t}, Q_{t}\right)$-tuple for some but not all $t$ in every open neighborhood of $t^{*}$, then $t^{*}$ is a critical value.

Moreover, there exists a $V$-critical event $\left(t^{*}, v\right)$ or a $F$ critical event $\left(t^{*}, m\right)$ such that $u$ contains $v$ or an edge with endpoint $v$, or $u$ is contained in the set of supports of $m$.

Proof. We can assume that there exists an open neighborhood $\mathcal{N}=\left(t_{0}, t_{1}\right)$ of $t^{*}$ such that $u$ is a maximal $\left(P_{t}, Q_{t}\right)$ tuple for $t=t^{*}$ or all $t \in\left(t^{*}, t_{1}\right)$ (or by symmetry for all $\left.t \in\left(t_{0}, t^{*}\right)\right)$, but is not a maximal $\left(P_{t}, Q_{t}\right)$-tuple for all $t \in \mathcal{N}$. Indeed, suppose that Lemma 5 is proved if $u$ satisfies this additional assumption. If $u$ does not satisfy this additional assumption, then there exists, in every open neighborhood of $t^{*}$, infinitely many maximal intervals (open or closed and possibly of zero length) in which either $u$ is a maximal $\left(P_{t}, Q_{t}\right)$-tuple for all $t$ or $u$ is not a maximal $\left(P_{t}, Q_{t}\right)$-tuple for all $t$. However, in this case, our assumption that Lemma 5 holds if $u$ satisfies the above additional

\footnotetext{
${ }^{8}$ For simplicity, we prove Lemma 5 under the assumptions that $u$ is maximal and distinct from $u_{e}$; note however that the lemma remains correct without these assumptions.
} 
assumption yields that each endpoint of these intervals is a critical value, contradicting Lemma 4.

We can also assume that $u$ contains no vertex $v$, or edge with endpoint $v$, such that $v$ lies on $\Pi_{t^{*}} \backslash l_{e}$ because otherwise $\left(t^{*}, v\right)$ is a $\mathrm{V}$-critical event such that $u$ contains $v$ or an edge with endpoint $v$, which concludes the proof.

We prove a series of claims that yields the result. Indeed, we prove the existence of a line $m$ in $\Pi_{t^{*}}$ whose set of supports contains $u$ (Claim C) such that (i) $m$ lies in a plane $\Psi \neq \Pi_{t^{*}}$ containing a face of $\mathbf{P}$ (Claim D), and (ii) $m$ is tangent in $\Psi$ to polygon $\mathbf{Q} \cap \Psi$ or $\mathbf{P} \cap \Psi$, at some point not on $l_{e}$ (Claim E). This proves that $\Pi_{t^{*}}$ contains a line $m$ whose set of supports contains $u$ and such that $\left(t^{*}, m\right)$ is an F-critical event, which concludes the proof.

We only consider in the following supports in $\mathbf{P}$ and in $\mathbf{Q}$; polytope $\mathbf{R}$ plays no role. We start by proving two preliminary facts.

Claim A. Each support in $u$ intersects $\Pi_{t}$ in exactly one point (possibly on $\left.l_{e}\right)$, for all $t$ in any sufficiently small open neighborhood $\mathcal{N}$ of $t^{*}$.

Moreover, the union of all supports in $u$ intersects $\Pi_{t}$ in at least two distinct points for all $t \neq t^{*}$ in $\mathcal{N}$. This property also holds for $t=t^{*}$ if $u$ is a maximal $\left(P_{t^{*}}, Q_{t^{*}}\right)$-tuple.

Proof of Claim A. Since $u$ is a $\left(P_{t}, Q_{t}\right)$-tuple for some $t$ in every open neighborhood of $t^{*}$, each support in $u$ intersects $\Pi_{t}$ for some $t$ in every open neighborhood of $t^{*}$. It thus follows from the assumption that $u$ contains no vertex $v$, or edge with endpoint $v$, such that $v$ lies on $\Pi_{t^{*}} \backslash l_{e}$ that each support in $u$ intersects $\Pi_{t}$ for all $t$ in any sufficiently small open neighborhood $\mathcal{N}$ of $t^{*}$. It follows that each support in $u$ either lies in $l_{e}$ or intersects $\Pi_{t}$ in exactly one point for all $t \in \mathcal{N}$. However, no edge of $u$ lies in $l_{e}$ because otherwise, if $x$ denotes such an edge of, say, $\mathbf{P}$, then any line tangent to $P_{t}$ in $\Pi_{t}$ and intersecting $x$ contains an endpoint of $x$ which is a vertex of $\mathbf{P}$; thus, by definition, $u$ does not contain $x$ but one of its endpoints. Hence each support of $u$ intersects $\Pi_{t}$ in exactly one point for all $t \in \mathcal{N}$.

We now prove that the union of the supports in $u$ intersects $\Pi_{t}$ in at least two distinct points for any $t \in \mathcal{N}$ such that $u$ is a maximal $\left(P_{t}, Q_{t}\right)$-tuple. Suppose for a contradiction that the union of the supports in $u$ intersects $\Pi_{t}$ in one single point $v$ for some $t \in \mathcal{N}$ such that $u$ is a maximal $\left(P_{t}, Q_{t}\right)$-tuple. Then polygons $P_{t}$ and $Q_{t}$ are both reduced to point $v$ because otherwise $u$ is not maximal (indeed a line in $\Pi_{t}$ tangent to $P_{t}$ and $Q_{t}$ at $v$ can be rotated about $v$ until it becomes tangent to $P_{t}$ or $Q_{t}$ in some other points). Thus $v$ is a vertex of $\mathbf{P}$ and of $\mathbf{Q}$ because the polytopes have non-empty interior. It follows that $v$ is a support in $u$. Thus vertex $v$ lies on $l_{e}$ since each support in $u$ intersects $\Pi_{t}$ for all $t \in \mathcal{N}$. Moreover, since $P_{t}$ and $Q_{t}$ are both reduced to point $v$, the set of supports of $l_{e}$ is $u$, contradicting the hypotheses of Lemma 5 .

Thus, if $u$ is a maximal $\left(P_{t}, Q_{t}\right)$-tuple for all $t \in\left(t^{*}, t_{1}\right)$, the union of the supports in $u$ intersects $\Pi_{t}$ in at least two distinct points for all $t \in\left(t^{*}, t_{1}\right)$ and thus for all $t \neq t^{*}$ in any sufficiently small open neighborhood of $t^{*}$. Also, if $u$ is a maximal $\left(P_{t}, Q_{t}\right)$-tuple for $t=t^{*}$, the union of the supports in $u$ intersects $\Pi_{t}$ in at least two distinct points for $t=t^{*}$ and thus for all $t$ in any sufficiently small open neighborhood of $t^{*}$.

Claim B. If $u$ is a maximal $\left(P_{t^{*}}, Q_{t^{*}}\right)$-tuple then $u$ consists of at least three supports.

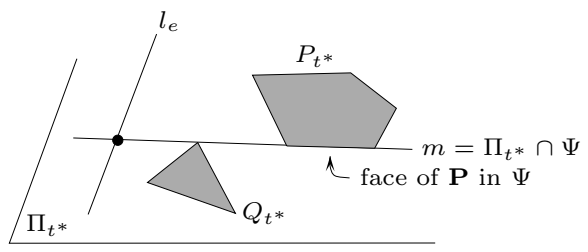

Figure 5: $m$ is tangent to $\mathbf{P}$ along a face in plane $\Psi \neq \Pi_{t^{*}}$.

Proof of Claim B. Suppose for a contradiction that $u$ consists of only two supports. It follows from Claim A that they intersect $\Pi_{t}$ in exactly two distinct points for all $t$ in any sufficiently small open neighborhood $\mathcal{N}$ of $t^{*}$. Thus there exists for all $t \in \mathcal{N}$ a unique line $m_{t}$ in $\Pi_{t}$ whose set of supports contains $u$; moreover $m_{t}$ is continuous in terms of $t$. Since $u$ is a $\left(P_{t^{*}}, Q_{t^{*}}\right)$-tuple, the set of supports of $m_{t^{*}}$ is $u$. Thus, for all $t$ in any sufficiently small $\mathcal{N}$, the set of supports of $m_{t}$ is $u$. It follows, since $m_{t^{*}}$ is tangent to $P_{t^{*}}$ and $Q_{t^{*}}$, that $m_{t}$ is tangent to $P_{t}$ and $Q_{t}$ for all $t \in \mathcal{N}$.

Hence, for all $t \in \mathcal{N}$, line $m_{t}$, whose set of supports is $u$, is tangent to $P_{t}$ and $Q_{t}$ in $\Pi_{t}$. Thus $u$ is a $\left(P_{t}, Q_{t}\right)$-tuple for all $t \in \mathcal{N}$. Moreover, $m_{t}$ is the unique line in $\Pi_{t}$ whose set of supports contains $u$, thus $u$ is a maximal $\left(P_{t}, Q_{t}\right)$-tuple for all $t \in \mathcal{N}$, contradicting the hypotheses of the lemma. $\square$

Claim C. There exists a line $m$ in $\Pi_{t^{*}}$ whose set of supports contains $u$ that is tangent to $P_{t^{*}}$ and $Q_{t^{*}}$ along an edge of one of them, say of $P_{t^{*}}$.

Proof of Claim C. Consider first the case where $u$ is a maximal $\left(P_{t^{*}}, Q_{t^{*}}\right)$-tuple. There exists in $\Pi_{t^{*}}$ a line $m$ tangent to $P_{t^{*}}$ and $Q_{t^{*}}$ whose set of supports is $u$. By Claim B, the set $u$ of supports of $m$ contains at least two supports in $\mathbf{P}$ (or in $\mathbf{Q}$ ). Furthermore, the supports of $m$ in one polytope intersect $\Pi_{t^{*}}$ in distinct points (by definition of supports). Thus $m$ intersects $P_{t^{*}}$ (or $Q_{t^{*}}$ ) in at least two distinct points and is tangent to $P_{t^{*}}$ and $Q_{t^{*}}$. The result follows since $P_{t^{*}}$ (and $Q_{t^{*}}$ ) is convex.

Consider now the case where $u$ is a maximal $\left(P_{t}, Q_{t}\right)$-tuple for all $t \in\left(t^{*}, t_{1}\right)$. Then, for all $t \in\left(t^{*}, t_{1}\right)$, there exists a line in $\Pi_{t}$ tangent to $P_{t}$ and $Q_{t}$ and whose set of supports is $u$. Moreover, by Claim $\mathrm{A}$, this line is unique for each $t \in\left(t^{*}, t_{1}\right)$ and varies continuously in terms of $t \in\left(t^{*}, t_{1}\right)$. When $t$ tends to $t^{*}$, the line tends to a line $m$ in $\Pi_{t^{*}}$ which is tangent to $P_{t^{*}}$ and $Q_{t^{*}}$ and whose set of supports contains $u$. If its set of supports strictly contains $u$ then $m$ is tangent to $P_{t^{*}}$ and $Q_{t^{*}}$ along an edge of one of them because the polygons are convex. Otherwise, $u$ is a $\left(P_{t^{*}}, Q_{t^{*}}\right)$-tuple.

We can suppose that $u$ is a non-maximal $\left(P_{t^{*}}, Q_{t^{*}}\right)$-tuple since we already treated the case where $u$ is maximal. There exists in $\Pi_{t^{*}}$ a line tangent to $P_{t^{*}}$ and $Q_{t^{*}}$ whose set of supports is $u$. Since $u$ is non-maximal this line is tangent to $P_{t^{*}}$ and $Q_{t^{*}}$ at a shared vertex, and can be rotated about this vertex in $\Pi_{t^{*}}$ until it gets tangent to $P_{t^{*}}$ and $Q_{t^{*}}$ in some other points; let $m$ denote the resulting line. The set of supports of $m$ contains $u$ and $m$ is tangent to $P_{t^{*}}$ and $Q_{t^{*}}$ along an edge of one of them because the polygons are convex.

Claim D. Line $m$ lies in a plane $\Psi \neq \Pi_{t^{*}}$ containing a face of $\mathbf{P}$.

Proof of Claim D. By Claim C, $m$ contains an edge of $P_{t^{*}}$; see Figure 5. This edge either intersects the relative 


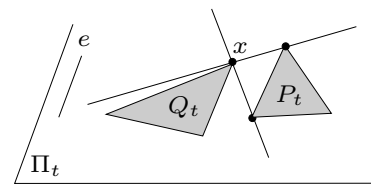

(a)

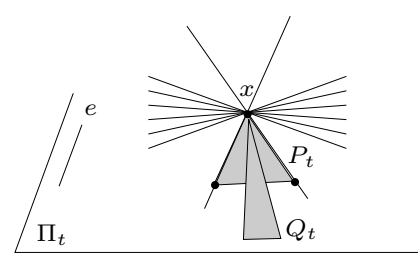

(b)
Figure 6: Lines through $x$ in $\Pi_{t}$ and tangent to $P_{t}$ and $\mathbf{Q}_{t}$.

interior of some face of $\mathbf{P}$ in which case we take $\Psi$ to be the plane containing that face, or it is an edge of $\mathbf{P}$ in which case we take $\Psi$ to be a plane, different from $\Pi_{t^{*}}$, containing one of the two faces of $\mathbf{P}$ incident to that edge.

Claim E. Line $m$ is tangent to $\mathbf{P} \cap \Psi$ or to $\mathbf{Q} \cap \Psi$, at some point not on $l_{e}$.

Proof of Claim E. By Claim C, $m$ is tangent to $Q_{t^{*}}$ and thus to $\mathbf{Q}$. It follows that $m$ is tangent to $\mathbf{Q} \cap \Psi$ or properly intersects it, in which case $\mathbf{Q} \cap \Psi$ is a face or an edge of $\mathbf{Q}$. If $m$ is not tangent to $\mathbf{Q} \cap \Psi$ at some point not on $l_{e}$ then $m$ is tangent to $\mathbf{Q} \cap \Psi$ only on $l_{e}$, or $\mathbf{Q} \cap \Psi$ is a face or an edge of $\mathbf{Q}$. These two situations are not generic since two edges of two distinct polytopes are then coplanar (in the first case an edge of $\mathbf{Q}$ and $e$ are coplanar, and in the later case a face of $\mathbf{P}$ is coplanar with a face or an edge of $\mathbf{Q}$ ). Hence proving this claim is straightforward under some general position assumption, however for polytopes in arbitrary position the proof is rather intricate and thus postponed to the appendix for the sake of clarity. $\square$

This concludes the proof of Lemma 5.

Let $\mathcal{I}$ be any interval of $\mathbb{R} / \pi \mathbb{Z}$ (open or closed, reduced to a point or not).

Lemma 6. Any edge or vertex of $\mathbf{P}$ or $\mathbf{Q}$ is in at most 2 $\mathbf{P Q}$-tuples that are maximal $\left(P_{t}, Q_{t}\right)$-tuples for all $t$ in $\mathcal{I}$.

Proof. Let $\tilde{t} \in \mathcal{I}$ and $x$ be an edge or vertex of $\mathbf{P}$ or $\mathbf{Q}$. If $x$ does not intersect $\Pi_{\tilde{t}}$ then no $\left(P_{\tilde{t}}, Q_{\tilde{t}}\right)$-tuple contains $x$. If $x$ intersects $\Pi_{\tilde{t}}$ in one point then there is, in general, at most two lines in $\Pi_{\tilde{t}}$ going through $x$ and tangent to $P_{\tilde{t}}$ and $Q_{\tilde{t}}$ (see Figure $\left.6(\mathrm{a})\right)$; in all cases there are at most $3\left(P_{\tilde{t}}, Q_{\tilde{t}}\right)$ tuples containing $x$ (see Figure 6(b)), however at most 2 of them are maximal. If $x$ intersects $\Pi_{\tilde{t}}$ in more than one point, $x$ is an edge lying in $\Pi_{\tilde{t}}$. Then any line in $\Pi_{\tilde{t}}$ intersecting $x$ and tangent to $P_{\tilde{t}}$ and $Q_{\tilde{t}}$ contains an endpoint of $x$ and thus $x$ belongs to no $\left(P_{\tilde{t}}, Q_{\tilde{t}}\right)$-tuple.

Hence at most 2 PQ-tuples contain $x$ and are maximal $\left(P_{t}, Q_{t}\right)$-tuples for $t=\tilde{t}$ and thus for all $t$ in $\mathcal{I}$.

Lemma 7. There are at most $O(p+q+r)$ PQR-tuples.

Proof. In order to count the number of distinct $\left(P_{t}, Q_{t}\right.$, $\left.R_{t}\right)$-tuples, we charge each maximal $\left(P_{t}, Q_{t}, R_{t}\right)$-tuple to a critical event. We then show that each critical event is charged at most a constant number of times. It then follows from Lemma 4 that there are $O(p+q+r)$ distinct maximal $\left(P_{t}, Q_{t}, R_{t}\right)$-tuples. A maximal $\left(P_{t}, Q_{t}, R_{t}\right)$-tuple consists of at most two supports in $\mathbf{P}$, at most three supports in $\mathbf{Q}$, and at most two supports in $\mathbf{R}$, and thus contains at most $3 \times 7 \times 3$ distinct subsets with at least one support in each of
$\mathbf{P}, \mathbf{Q}$ and $\mathbf{R}$. Each maximal $\left(P_{t}, Q_{t}, R_{t}\right)$-tuple thus contains at most $3 \times 7 \times 3$ distinct $\left(P_{t}, Q_{t}, R_{t}\right)$-tuples which implies the result.

Let $s$ be a maximal $\left(P_{t}, Q_{t}, R_{t}\right)$-tuple and let $\mathcal{I}$ be a maximal connected subset of $\mathbb{R} / \pi \mathbb{Z}$ such that $s$ is a maximal $\left(P_{t}, Q_{t}, R_{t}\right)$-tuple for all $t \in \mathcal{I}$. Let $u$ be a maximal $\left(P_{t}, Q_{t}\right)$ tuple and $u^{\prime}$ a maximal $\left(Q_{t}, R_{t}\right)$-tuple whose union is $s$ and who share at least a support in $\mathbf{Q}$.

If $\mathcal{I}=\mathbb{R} / \pi \mathbb{Z}$ then $u$ is a maximal $\left(P_{t}, Q_{t}\right)$-tuple for all $t \in \mathcal{I}$. Since $u$ is maximal, the union of the supports in $u$ intersects $\Pi_{t}$ in at least two distinct points. Moreover, each support in $u$ intersects $\Pi_{t}$ for all $t \in \mathcal{I}$ and thus intersects $l_{e}$. It follows that $l_{e}$ is the only line in $\Pi_{t}$ whose set of supports is $u$, for any $t \in \mathcal{I}$. Similarly for $u^{\prime}$. Thus $s$ is the set of supports of $l_{e}$. We can thus assume in the following that $\mathcal{I} \neq \mathbb{R} / \pi \mathbb{Z}$, and only count the maximal $\left(P_{t}, Q_{t}, R_{t}\right)$-tuples that are not the set of support of $l_{e}$.

Interval $\mathcal{I}$ is thus a non-empty interval of $\mathbb{R} / \pi \mathbb{Z} ;$ it can be open or closed, reduced to a point or not. Let $w_{0}$ and $w_{1}$ denote its endpoints.

If $s$ contains a vertex $v$, or an edge with endpoint $v$, such that $v$ lies in $\Pi_{w_{i}} \backslash l_{e}$, for $i=0$ or 1 , then we charge $s$ to the $\mathrm{V}$-critical event $\left(w_{i}, v\right)$. Otherwise, we charge $s$ to a F-critical event $\left(w_{i}, m\right)$ where $m$ is a line in $\Pi_{w_{i}}$ whose set of supports contains $u$ or $u^{\prime}$. Such a V-critical or F-critical event exists by Lemma 5 .

We now prove that each critical event is charged by at most a constant number of distinct maximal $\left(P_{t}, Q_{t}, R_{t}\right)$ tuples. As mentioned before, that will imply the result.

Consider a $\mathrm{V}$-critical event $\left(t^{*}, v\right)$ that is charged by a maximal $\left(P_{t}, Q_{t}, R_{t}\right)$-tuple $s$. By the charging scheme, $s$ contains a support $x$ that is $v$ or an edge with endpoint $v$, and $s$ is a maximal $\left(P_{t}, Q_{t}, R_{t}\right)$-tuple for all $t$ in at least one of three intervals, $\left\{t^{*}\right\}$ and two open intervals having $t^{*}$ as endpoint; denote by $\mathcal{I}_{1}, \mathcal{I}_{2}, \mathcal{I}_{3}$ these intervals.

By Lemma 6, at most 2 PQ-tuples contain $x$ and are maximal $\left(P_{t}, Q_{t}\right)$-tuples for all $t$ in $\mathcal{I}_{i}$. Moreover, each of these PQ-tuples contains at most 2 supports in $\mathbf{Q}$, and each of these supports belongs to at most 2 QR-tuples that are maximal $\left(Q_{t}, R_{t}\right)$-tuples for all $t$ in $\mathcal{I}_{i}$. Thus at most 8 PQRtuples contain $x$ and are maximal $\left(P_{t}, Q_{t}, R_{t}\right)$-tuples for all $t$ in $\mathcal{I}_{i}$, for each $i=1, \ldots, 3$. Hence any $\mathrm{V}$-critical event $\left(t^{*}, v\right)$ is charged by at most 24 distinct maximal $\left(P_{t}, Q_{t}, R_{t}\right)$-tuples.

Consider now a F-critical event $\left(t^{*}, m\right)$ that is charged by a maximal $\left(P_{t}, Q_{t}, R_{t}\right)$-tuple $s$, and define as before $u$ and $u^{\prime}$. By the charging scheme, the set of supports of $m$ contains $u$ or $u^{\prime}$ (or both); suppose without loss of generality that it contains $u$. The set of supports of $m$ contains at most two supports in $\mathbf{P}$ and at most two supports in $\mathbf{Q}$. Since $u$ contains at least one support in $\mathbf{P}$ and at least one support in $\mathbf{Q}$, there are at most $3^{2}$ choices for $u$.

By the charging scheme, $s$ is a maximal $\left(P_{t}, Q_{t}, R_{t}\right)$-tuple for all $t$ in at least one of 3 intervals, $\left\{t^{*}\right\}$ and two open intervals having $t^{*}$ as endpoint; denote by $\mathcal{I}_{1}, \mathcal{I}_{2}, \mathcal{I}_{3}$ these intervals. It follows from Lemma 6 that, for each support $x$ of $\mathbf{Q}$ in $u$, at most $2 \mathbf{Q R}$-tuples contain $x$ and are maximal $\left(Q_{t}, R_{t}\right)$-tuples for all $t$ in $\mathcal{I}_{i}$. There are at most $3^{2}$ choices for $u, 2$ for $x, 3$ for $i$ and 2 for the QR-tuples containing $x$. Hence any F-critical event $\left(t^{*}, m\right)$ is charged by at most $2^{2} \times 3^{3}$ distinct maximal $\left(P_{t}, Q_{t}, R_{t}\right)$-tuples.

Therefore each critical event is charged by at most a constant number of distinct maximal $\left(P_{t}, Q_{t}, R_{t}\right)$-tuples, which concludes the proof. 
We now show in the next two lemmas that there are at most $O(p+q+r)$ connected components of generic tangent lines.

LEMMA 8. The set of transversals to $e$ and to the edges and vertices of a PQR-tuple contains at most $O(1)$ connected components of tangents to $\mathbf{P}, \mathbf{Q}, \mathbf{R}$ and $\mathbf{S}$.

Proof. The set of transversals to $\mu$ segments (possibly of zero length) consists of at most $\mu$ connected components [4]. Since the union of $\{e\}$ and any PQR-tuple consists of at most 8 segments (possibly of zero length), the result follows if the set of transversals to $e$ and to the edges and vertices of a PQR-tuple is finite. We omit here the proof in the case where this set of transversals is not finite.

Proposition 9. The generic tangent lines form $O(p+$ $q+r)$ connected components.

Proof. A generic tangent line is transversal to $e$ and to the edges and vertices of a PQR-tuple, by definition and Lemma 3. Moreover, there are $O(p+q+r)$ PQR-tuples by Lemma 7. The result thus follows from Lemma 8.

\section{Nongeneric tangent lines}

We count here the number of nongeneric tangent lines. Note that, as mentioned before, there are no such lines under some adequate general position assumption.

We first introduce some notation. A plane $\Pi_{t}$ is called a tangent plane if and only if it is tangent to $\mathbf{P}, \mathbf{Q}$, or $\mathbf{R}$ and at least one point of tangency does not lie on $l_{e}$.

Lemma 10. A nongeneric tangent line lies in a tangent plane or properly intersects an edge of $\mathbf{P}, \mathbf{Q}$, or $\mathbf{R}$ lying in $l_{e}$.

Proof. Let $\ell$ be a nongeneric tangent line. By definition, $\ell$ properly intersects $P_{t}, Q_{t}$, or $R_{t}$ in some plane $\Pi_{t}$. Suppose without loss of generality that $\ell$ properly intersects $P_{t}$. Since $\ell$ is tangent to $\mathbf{P}, P_{t}$ is a face or an edge of $\mathbf{P}$ which implies that $\Pi_{t}$ is tangent to $\mathbf{P}$. Thus $\Pi_{t}$ is a tangent plane or $P_{t}$ is an edge contained in $l_{e}$. In the first case, $\ell$ lies in the tangent plane $\Pi_{t}$. In the latter case, $\ell$ properly intersects an edge of $\mathbf{P}$ lying in $l_{e}$.

We count first the nongeneric tangent lines lying in tangent planes and then the ones that properly intersect an edge of $\mathbf{P}, \mathbf{Q}$, or $\mathbf{R}$ lying in $l_{e}$.

Lemma 11. The nongeneric tangent lines lying in all tangent planes form $O(p+q+r)$ connected components.

Proof. We first consider the nongeneric tangent lines lying in a given tangent plane $\Pi_{t}$. Such lines intersect, properly or not, $e, P_{t}, Q_{t}$, and $R_{t}$ in the plane $\Pi_{t}$. Let $\ell$ denote such a line and $\mathcal{A}_{p}$ (resp. $\left.\mathcal{A}_{t}\right)$ the subset of $\left\{e, P_{t}, Q_{t}, R_{t}\right\}$ of polygons that properly intersect $\ell$ (resp. are tangent to $\ell)$. We can move $\ell$ in $\Pi_{t}$ into a line tangent to at least two polygons of $\left\{e, P_{t}, Q_{t}, R_{t}\right\}$, while intersecting (properly ot not) the polygons of $\mathcal{A}_{p}$ and remaining tangent to the polygons of $\mathcal{A}_{t}$ during the motion. There are $O(p+q+r)$ such tangents, thus the nongeneric tangent lines lying in $\Pi_{t}$ form $O(p+q+r)$ connected components.

The result follows since there are at most 2 tangent planes per polytope.
LEMma 12. The nongeneric tangent lines that properly intersect an edge of $\mathbf{P}, \mathbf{Q}$, or $\mathbf{R}$ lying in $l_{e}$ form $O(p+q+r)$ connected components.

Proof. Let $\ell$ denote such a line. Let $o_{p}$ be the union of edges of $\mathbf{P}$ that are contained in $l_{e}$; if no such edge exists, $o_{p}$ is empty. We define $o_{q}$ and $o_{r}$ similarly. Line $\ell$ intersects at least one of $o_{p}, o_{q}$, and $o_{r}$. Let $\alpha=1,2$, or 3 be the number of these sets intersected by $\ell$. We consider in turn the three cases depending on the value of $\alpha$.

Case $\boldsymbol{\alpha}=3$. Sets $o_{p}, o_{q}$, and $o_{r}$ are all nonempty since $\ell$ intersects each of them. If $o_{p}, o_{q}, o_{r}$ and $e$ do not all pairwise intersect, i.e., if $I=e \cap o_{p} \cap o_{q} \cap o_{r}$ is empty then there is exactly one line $\ell$ intersecting all four $o_{p}, o_{q}, o_{r}$ and $e$, and that is line $l_{e}$. If on the contrary, $I$ is nonempty, then the nongeneric tangent lines intersecting $I$ are the line intersecting $I$ and not intersecting the interior of $\mathbf{P}, \mathbf{Q}, \mathbf{R}$ and $\mathbf{S}$. These lines thus form one connected component.

Case $\boldsymbol{\alpha}=2$. Suppose that $\ell$ intersects $o_{p}$ and $o_{q}$; the other cases are similar. Then $o_{p}$ and $o_{q}$ are nonempty. Again if $I=e \cap o_{p} \cap o_{q}$ is empty, then there is at most one line $\ell=l_{e}$ intersecting $e, o_{p}$, and $o_{q}$ and tangent to $\mathbf{R}$. If on the contrary, $I$ is nonempty then there are at most $r$ edges of $\mathbf{R}$ that may induce some tangents with $I$. By Lemma 8, that implies that there are at most $O(r)$ connected components of lines intersecting $e, o_{p}$, and $o_{q}$, and tangent to $\mathbf{P}, \mathbf{Q}, \mathbf{R}$ and $\mathbf{S}$.

Case $\boldsymbol{\alpha}=1$. Suppose that $\ell$ intersects $o_{p}$; the other cases are similar. Then $o_{p}$ is nonempty. Again if $I=e \cap o_{p}$ is empty, then there is at most one line $\ell=l_{e}$ intersecting both $o_{p}$ and $e$ and tangent to polytopes $\mathbf{Q}$ and $\mathbf{R}$. Consider now the case where the common intersection $I$ is nonempty. Lemmas 9, 10 and 11, when applied to $I$ and the three polytopes $\mathbf{Q}, \mathbf{R}$, and again $\mathbf{R}$ yields that there are at most $O(q+r)$ connected components of lines intersecting $I$ and tangent to $\mathbf{P}, \mathbf{Q}, \mathbf{R}$ and $\mathbf{S}$.

Lemmas 10, 11 and 12 yield the following result.

LEMMA 13. There are at most $O(p+q+r)$ connected components of nongeneric tangent lines.

This result together with Lemma 9 concludes the proof of the Main Lemma.

\section{UPPER BOUND FOR THEOREM 2}

We prove in this section the upper bound of Theorem 2 on the number of tangents to any 4 among $k$ polytopes. The lower bound is proved in Section 4

Consider $k$ polytopes $\mathbf{P}_{1}, \ldots, \mathbf{P}_{k}$ with $n_{1}, \ldots, n_{k}$ edges respectively. Let $\mathbf{P}_{i}, \mathbf{P}_{j}, \mathbf{P}_{l}$, and $\mathbf{P}_{m}$ be four pairwise distinct polytopes and $e$ an edge of $\mathbf{P}_{i}$. From the Main Lemma, we know that the number of connected components of lines intersecting $e$ and tangent to $\mathbf{P}_{i}, \mathbf{P}_{j}, \mathbf{P}_{l}$, and $\mathbf{P}_{m}$ is no more than $C\left(n_{j}+n_{l}+n_{m}\right)$, where $C$ is some constant. There are $n$ edges in the scene, so the number $T$ of connected components of tangents to four polytopes satisfies

$$
T \leqslant n \sum_{j<l<m} C\left(n_{j}+n_{l}+n_{m}\right) .
$$

Since each $n_{i}, 1 \leqslant i \leqslant k$, appears $\left(\begin{array}{c}k-1 \\ 2\end{array}\right)$ times in the sum,

$$
T \leqslant C n \sum_{1 \leqslant i \leqslant k} n_{i}\left(\begin{array}{c}
k-1 \\
2
\end{array}\right)=C n^{2}\left(\begin{array}{c}
k-1 \\
2
\end{array}\right)
$$

so $T$ is $O\left(n^{2} k^{2}\right)$ as claimed. 


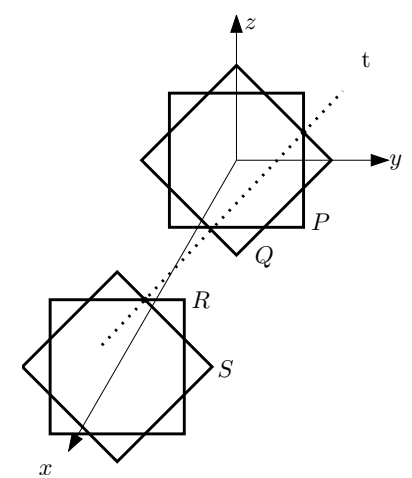

Figure 7: Lower bound example for Lemma 14.

\section{LOWER BOUNDS FOR THEOREMS 1 AND 2}

We provide in this section the lower bounds needed for Theorems 1 and 2 .

LEMMA 14. There exist four disjoint polytopes of complexity $O(n)$ such that the number of common tangent lines is finite and $\Omega\left(n^{2}\right)$. There also exist two polytopes of complexity $O(n)$ and two polytopes of complexity $O(m)$ such that the number of common tangent lines is finite and $\Omega(m n)$.

Proof. We consider four planar regular polygons $P, Q$, $R$, and $S$, each with $n$ vertices, embedded in $\mathbb{R}^{3} . P$ is centered at the origin and parallel to the $y z$-plane, $Q$ is obtained from $P$ by a rotation of angle $\frac{\pi}{n}$ about the $x$-axis, and $R$ and $S$ are obtained from $P$ and $Q$, respectively, by a translation of length 1 in the positive $x$-direction (see Figure 7 ). We transform the polygons $P$ and $Q$ into the polytopes $\mathbf{P}$ and $\mathbf{Q}$ by adding a vertex at coordinates $(\varepsilon, 0,0)$. Similarly, we transform the polygons $R$ and $S$ into the polytopes $\mathbf{R}$ and $\mathbf{S}$ by adding a vertex at coordinates $(1+\varepsilon, 0,0)$.

For $\varepsilon$ sufficiently small, the lines tangent to $\mathbf{P}, \mathbf{Q}, \mathbf{R}$ and $\mathbf{S}$ are the lines through a vertex of $P \cap Q$ and a vertex of $R \cap S$. Since $P \cap Q$ and $R \cap S$ have $2 n$ vertices each, there are $4 n^{2}$ tangent lines. Now, moving $\mathbf{P}$ and $\mathbf{S}$ by $2 \varepsilon$ in the $x$ direction ensures the disjointness of the polytopes while preserving the existence of the tangents if $\varepsilon$ is small enough.

Replacing $R$ and $S$ in the above construction by regular polygons each with $m$ vertices yields the $\Omega(m n)$ lower bound in the case of two polytopes of complexity $O(n)$ and two polytopes of complexity $O(m)$.

We now provide the lower bound needed for Theorem 2 .

LEMma 15. There exist $k$ disjoint polytopes of total complexity $O(n)$ such that the number of non-occluded maximal line segments tangent to four of them is finite and $\Omega\left(k^{2} n^{2}\right)$.

Proof. The lower bound example is similar to the one with four polyhedra. For simplicity suppose that $n$ and $k$ are such that $\frac{n}{k}$ and $\frac{k}{4}$ are integers. We first take a $\frac{n}{k}$-regular polygon $A_{1}$ in the plane $x=0$. Next we consider a copy, $B_{0}$, of $A_{1}$ scaled by a factor of $(1+\varepsilon)$, and on each edge of $B_{0}$ we place $\frac{k}{4}$ points. Polygon $B_{i}, 1 \leqslant i \leqslant \frac{k}{4}$, is constructed by taking the $i^{\text {th }}$ point on each edge of $B_{0}$. If $\varepsilon$ is small enough, the intersection points of $A_{1}$ and $B_{i}$ are outside the other polygons $B_{j}$ for $1 \leqslant j \leqslant \frac{k}{4}$ and $i \neq j$. Now the $A_{i}$, for $2 \leqslant i \leqslant \frac{k}{4}$, are constructed as copies of $A_{1}$ scaled by a factor

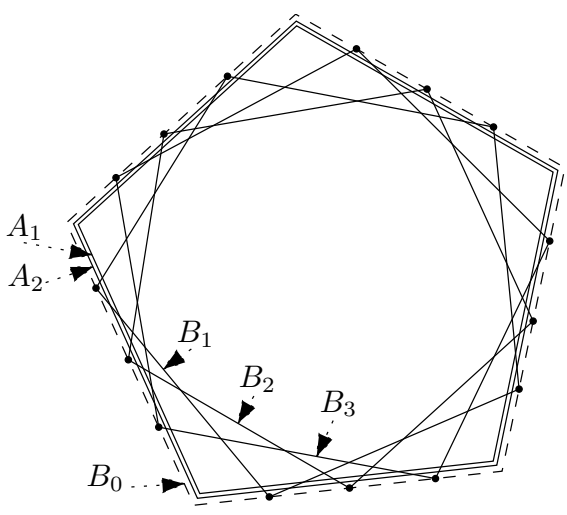

Figure 8: The polygons $A_{i}$ and $B_{i}$.

$1+\frac{i}{k} \varepsilon$ (see Figure 8). For the moment, all polygons lie in plane $x=0$. We now construct 4 families of $\frac{k}{4}$ polygons each:

- $P_{i}$ is a copy of $A_{i}$ translated by $i \epsilon$ in the negative $x$ direction

- $Q_{i}$ is a copy of $B_{i}$ translated by $i \epsilon$ in the positive $x$ direction

- $R_{i}$ is a copy of $B_{i}$ translated by $1-i \epsilon$ in the positive $x$ direction

- $S_{i}$ is a copy of $A_{i}$ translated by $1+i \epsilon$ in the positive $x$ direction

Any choice of four polygons, one in each family $P_{i}, Q_{j}$, $R_{l}$ and $S_{m}$, reproduces the quadratic example of Lemma 14 with polygons of size $\frac{n}{k}$ and thus with total number of tangents larger than $\left(\frac{k}{4}\right)^{4} 4\left(\frac{n}{k}\right)^{2}=\frac{n^{2} k^{2}}{4}$. Furthermore the lines tangent to $P_{i}, Q_{j}, R_{l}$ and $S_{m}$ are only occluded by $P_{i^{\prime}}$ and $S_{m^{\prime}}$ for $i^{\prime}>i$ and $m^{\prime}>m$, that is, beyond the portion of the tangents containing the contact points. The $k$ polygons can be transformed into $k$ convex polyhedra as in Lemma 14 .

\section{REFERENCES}

[1] P. K. Agarwal. On stabbing lines for convex polyhedra in 3D. Comput. Geom.: Theory and Appl., 4(4):177-189, 1994.

[2] M. de Berg, D. Halperin, M. Overmars, and M. van Kreveld, Sparse arrangements and the number of views of polyhedral scenes, In Internat. J. Comput. Geom. Appl. 7(3):175-195, 1997.

[3] H. Brönnimann, O. Devillers, V. Dujmovic, H. Everett, M. Glisse, X. Goaoc, S. Lazard, H.-S. Na, and S. Whitesides, On the Number of Lines Tangent to Four Convex Polyhedra. In Proc. Can. Conf. Comp. Geom., pp. 113-117, 2002.

[4] H. Brönnimann, H. Everett, S. Lazard, F. Sottile, and S. Whitesides. The number of transversals to line segments in $\mathbb{R}^{3}$. In Proc. Can. Conf. Comp. Geom., Halifax, 2003. Also INRIA Technical report No. 4864 , July 2003.

[5] O. Devillers, V. Dujmovic, H. Everett, X. Goaoc, S. Lazard, H.-S. Na, and S. Petitjean. The expected number of $3 \mathrm{D}$ visibility events is linear. SIAM J. Comput., 32(6):1586-1620, June 2003.

[6] F. Durand. 3D Visibility: Analytical study and Applications. PhD Thesis, Université Joseph Fourier, Grenoble, France, July 1999. 


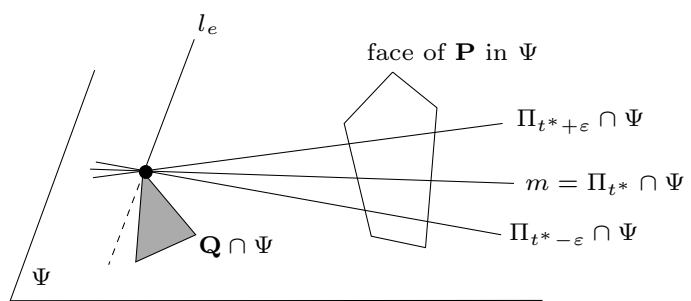

(a)

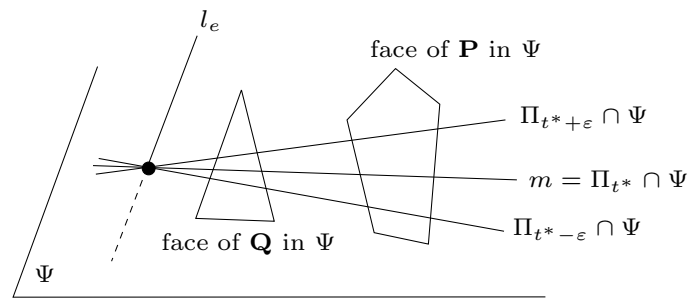

(b)

Figure 9: $m$ is tangent to $\mathbf{P}$ along a face in $\Psi$ and (a) to $\mathrm{Q} \cap \Psi$ only on $l_{e}$ or (b) to $\mathrm{Q}$ along a face in $\Psi$.

[7] F. Durand. A multidisciplinary survey of visibility. ACM Siggraph Course Notes: Visibility, Problems, Techniques, and Applications, 2000.

[8] F. Durand, G. Drettakis, and C. Puech. The visibility skeleton: a powerful and efficient multi-purpose global visibility tool. In Comput. Graphics Proc. Ann. Conf. Series (SIGGRAPH) 31:89-100, 1997.

[9] F. Durand, G. Drettakis, and C. Puech. The 3D visibility complex. ACM Trans. on Graphics, 21(2):176-206, 2002.

[10] A. Efrat, L. Guibas, O. Hall-Holt, and L. Zhang. On Incremental Rendering of Silhouette Maps of a Polyhedral Scene. In Proc. 11th ACM-SIAM Symp. on Discrete Algorithms, pp. 910-917, 2000.

[11] M. Pocchiola and G. Vegter. The visibility complex. Internat. J. Comput. Geom. Appl., 6(3):279-308, 1996.

[12] R. Schiffenbauer. A Survey of Aspect Graphs. Technical Report TR-CIS-2001-01, Dept. of Comp. and Info. Science, Polytechnic U., 2001.

\section{APPENDIX}

\section{Proof of Claim E}

We give here a complete proof of Claim E of Lemma 5 when the polytopes are in arbitrary position. We first recall the claim.

Claim E. Line $m$ is tangent to $\mathbf{P} \cap \Psi$ or to $\mathbf{Q} \cap \Psi$, at some point not on $l_{e}$.

In the following, we keep all the notation and assumptions made in the proof of Lemma 5 and all the claims that are referred to are those of Lemma 5 .

We assume for a contradiction that line $m$ does not satisfy Claim E, i.e., $m$ is not tangent to $\mathbf{P} \cap \Psi$ or to $\mathbf{Q} \cap \Psi$ at any point other than on $l_{e}$. We prove that the set of supports of $m$ is $u$ and is a maximal $\left(P_{t}, Q_{t}\right)$-tuple for all $t$ in any sufficiently small neighborhood of $t^{*}$, contradicting the hypotheses of Lemma 5 .
Since $m$ is tangent to $\mathbf{Q}$ (by Claim $\mathbf{C}$ ), $m$ is tangent to $\mathbf{Q} \cap \Psi$ only on $l_{e}$ (see Figure $9(\mathrm{a})$ ), or $m$ properly intersects $\mathbf{Q} \cap \Psi$ which is then a face or an edge of $\mathbf{Q}$ (see Figure $9(\mathrm{~b})$ ). Similarly $m$ is tangent to $\mathbf{P} \cap \Psi$ only on $l_{e}$, or $m$ properly intersects it; however $\mathbf{P} \cap \Psi$ is necessarily a face of $\mathbf{P}$ by Claim D.

Let $m_{t}$ be the line $\Psi \cap \Pi_{t}$ for all $t$ in any sufficiently small open neighborhood $\mathcal{N}$ of $t^{*}$; line $m_{t}$ is well defined since $\Psi \cap \Pi_{t^{*}}$ is line $m$ by Claims C and D. Recall that we only consider here supports in $\mathbf{P}$ and $\mathbf{Q}$.

LEMMA 16. The set of supports of $m_{t}$ is $u$ for some $t$ in any sufficiently small open neighborhood $\mathcal{N}$ of $t^{*}$.

Proof. We first prove that the supports in $u$ are supports of $m_{t}$ for all $t \in \mathcal{N}$. A support vertex in $u$ lies on $l_{e}$ by Claim A and thus lies in $\Pi_{t}$ for all $t$. A support vertex in $u$ also lies on $m$ by Claim $\mathrm{C}$ and thus lies in plane $\Psi$ by Claim D. Hence, for all $t \in \mathcal{N}$, the support vertices in $u$ lie on $m_{t}$, and thus are supports of $m_{t}$.

In order to prove that the support edges in $u$ are supports of $m_{t}$, it is sufficient (by Claim C) to prove that the support edges of $m$ are supports of $m_{t}$. The support edges of $m$ in $\mathbf{P}$ lie in plane $\Psi$ (see Figure $9(\mathrm{~b})$ ) because $\Psi$ contains $m$ and a face of $\mathbf{P}$ (indeed if $m$ intersects an edge of $\mathbf{P}$ not in $\Psi$ then $m$ contains one of its endpoints, and thus the edge is not a support). Thus all the support edges of $m$ lie in $\Psi$ and $m$ contains none of their endpoints (by definition). Since $m_{t}$ lies in $\Psi$ for all $t$ and $m_{t^{*}}=m$, line $m_{t}$ intersects all the support edges of $m$ and contains none of their endpoints for all $t$ in any sufficiently small open neighborhood $\mathcal{N}$ of $t^{*}$. Hence the support edges of $m$ in $\mathbf{P}$ are supports of $m_{t}$ for all $t \in \mathcal{N}$.

Consider the case where $\mathbf{Q} \cap \Psi$ is a face or an edge of $\mathbf{Q}$. Similarly as for $\mathbf{P}$, the support edges of $m$ in $\mathbf{Q}$ lie in plane $\Psi$, and thus are supports of $m_{t}$ for all $t \in \mathcal{N}$.

Consider now the case where $m$ is tangent to $\mathbf{Q} \cap \Psi$ only on $l_{e}$ at, say, point $v$ (see Figure $9(\mathrm{a})$ ). Then $v$ lies in $\Psi$ (since $m \subset \Psi$ by Claim D) and also lies in $\Pi_{t}$ for all $t$ (since $l_{e} \subset \Pi_{t}$ for all $t$ ). Hence $m_{t}$ contains $v$ for all $t \in \mathcal{N}$. Moreover, $m_{t}$ is tangent to $\mathbf{Q} \cap \Psi$ only at $v$ for all $t$ in any sufficiently small open neighborhood $\mathcal{N}$ of $t^{*}$. Hence the set of supports of $m_{t}$ in $\mathbf{Q}$ is invariant for all $t \in \mathcal{N}$.

We have so far proved that the set of supports of $m_{t}$ contains $u$ for all $t \in \mathcal{N}$.

We now prove that the set of supports of $m_{t}$ is $u$ for some $t \in \mathcal{N}$. Consider first the case where $u$ is a maximal $\left(P_{t^{*}}, Q_{t^{*}}\right)$-tuple. Then, by Claim A, the union of the supports in $u$ intersects $\Pi_{t^{*}}$ in at least two distinct points, thus $m_{t^{*}}=m$ is the only line in $\Pi_{t^{*}}$ whose set of supports contains $u$. Moreover, since $u$ is a $\left(P_{t^{*}}, Q_{t^{*}}\right)$-tuple, there exists a line in $\Pi_{t^{*}}$ whose set of supports is $u$. Hence the set of supports of $m_{t^{*}}$ is $u$.

Consider now the case where $u$ is a maximal $\left(P_{t}, Q_{t}\right)$-tuple for all $t \in\left(t^{*}, t_{1}\right)$. By Claim $\mathrm{A}$, for all $t \in\left(t^{*}, t_{1}\right)$, the union of the supports in $u$ intersects $\Pi_{t}$ in at least two distinct points, thus $m_{t}$ is the only line in $\Pi_{t}$ whose set of supports contains $u$. For all $t \in\left(t^{*}, t_{1}\right)$, since $u$ is a $\left(P_{t}, Q_{t}\right)$-tuple there exists a line in $\Pi_{t}$ whose set of supports is $u$. Hence the set of supports of $m_{t}$ is $u$ for all $t \in\left(t^{*}, t_{1}\right)$.

LEMMA 17. The set of supports of $m_{t}$ is invariant for $t$ ranging in any sufficiently small open neighborhood $\mathcal{N}$ of $t^{*}$. 
Proof. First if $m=l_{e}$ then $m_{t}=l_{e}$ for all $t \in \mathcal{N}$ because $\Psi$ contains $m=l_{e}$ (by Claim D) and $\Pi_{t}$ contains $l_{e}$ for all $t$ (by definition). Thus the set of supports of $m_{t}$ is invariant for all $t \in \mathcal{N}$. We now assume that $m \neq l_{e}$.

Line $m$ is tangent to polygon $P_{t^{*}}$ along an edge by Claim C. Thus $m$ is tangent to $\mathbf{P}$ in at least two points. Hence, since $\mathbf{P} \cap \Psi$ is a face of $\mathbf{P}$ and $m$ lies in $\Psi$, either $m$ properly intersects $\mathbf{P} \cap \Psi$ or $m$ is tangent to $\mathbf{P} \cap \Psi$ along one of its edges. In the later case, the edge does not lie in $l_{e}$ since $m \neq l_{e}$, thus $m$ is tangent to $\mathbf{P} \cap \Psi$ at some point not on $l_{e}$, contradicting our assumptions. Hence $m$ properly intersects the face of $\mathbf{P}$ in $\Psi$.

It follows that, if $m$ contains a vertex of $\mathbf{P}$, then this vertex is an endpoint of a support edge of $m_{t}$ for all $t$ in any sufficiently small open neighborhood of $t^{*}$ (indeed $m_{t}$ lies in $\Psi$ and tends to $m$ when $t$ tends to $\left.t^{*}\right)$. By Lemma 16, the set of supports of $m_{t}$ is $u$ for some $t$ in any sufficiently small open neighborhood of $t^{*}$. Hence, if $m$ contains a vertex of $\mathbf{P}$, this vertex is an endpoint of a support edge in $u$. By assumption $u$ contains no edge with endpoint on $\Pi_{t^{*}} \backslash l_{e}$, thus $m$ contains no vertex of $\mathbf{P}$ except possibly on $l_{e}$ (since $m$ lies in $\left.\Pi_{t^{*}}\right)$. It thus follows that the set of supports of $m_{t}$ in $\mathbf{P}$ is invariant for $t$ ranging in any sufficiently small open neighborhood of $t^{*}$ (since $m_{t} \subset \Psi$ tends to $m$ when $t$ tends to $t^{*}$ and all supports of $m$ lie in $\Psi$ ).

Now consider the case where $m$ properly intersects $\mathbf{Q} \cap \Psi$ which is a face or an edge of $\mathbf{Q}$. Similarly as for $\mathbf{P}, m$ contains no vertex of $\mathbf{Q}$ except possibly on $l_{e}$ and thus the set of supports of $m_{t}$ in $\mathbf{Q}$ is invariant for $t$ ranging in any sufficiently small open neighborhood of $t^{*}$.

Finally, consider the case where $m$ is tangent to $\mathbf{Q} \cap \Psi$ only on $l_{e}$. Then, as in the proof of Lemma 16, the set of supports of $m_{t}$ in $\mathbf{Q}$ is invariant for all $t$ ranging in any sufficiently small open neighborhood of $t^{*}$, which concludes the proof.

Proof of Claim E. It follows from Lemmas 16 and 17 that the set of supports of $m_{t}$ is invariant and equal to $u$ for all $t \in \mathcal{N}$. Moreover, since $m_{t}$ varies continuously with $t$ and $m=m_{t^{*}}$ is tangent to $P_{t^{*}}$ and $Q_{t^{*}}$ (by Claim C), line $m_{t}$ is tangent to $P_{t}$ and $Q_{t}$ for all $t \in \mathcal{N}$. Hence $u$ is a $\left(P_{t}, Q_{t}\right)$-tuple for all $t \in \mathcal{N}$. We now prove that $u$ is a maximal $\left(P_{t}, Q_{t}\right)$-tuple for all $t \in \mathcal{N}$.

As we have seen before, $m=m_{t^{*}}$ is tangent to $\mathbf{P}$ in at least two points (by Claim $\mathrm{C}$ ), thus $m_{t^{*}}$ intersects its supports in at least two distinct points. Moreover the set of supports of $m_{t^{*}}$ is $u$. Thus there is a unique line in $\Pi_{t^{*}}$ whose set of supports contains $u$. Hence $u$ is a maximal $\left(P_{t^{*}}, Q_{t^{*}}\right)$-tuple.

By Claim A, $m_{t}$ is the unique line in $\Pi_{t}$ whose set of supports contains $u$ for all $t \neq t^{*}$ in $\mathcal{N}$. Thus $u$ is a maximal $\left(P_{t}, Q_{t}\right)$-tuple for all $t \neq t^{*}$ in $\mathcal{N}$.

Hence $u$ is a maximal $\left(P_{t}, Q_{t}\right)$-tuple for all $t \in \mathcal{N}$, contradicting the hypotheses of Lemma 5 and thus concluding the proof of Claim E.
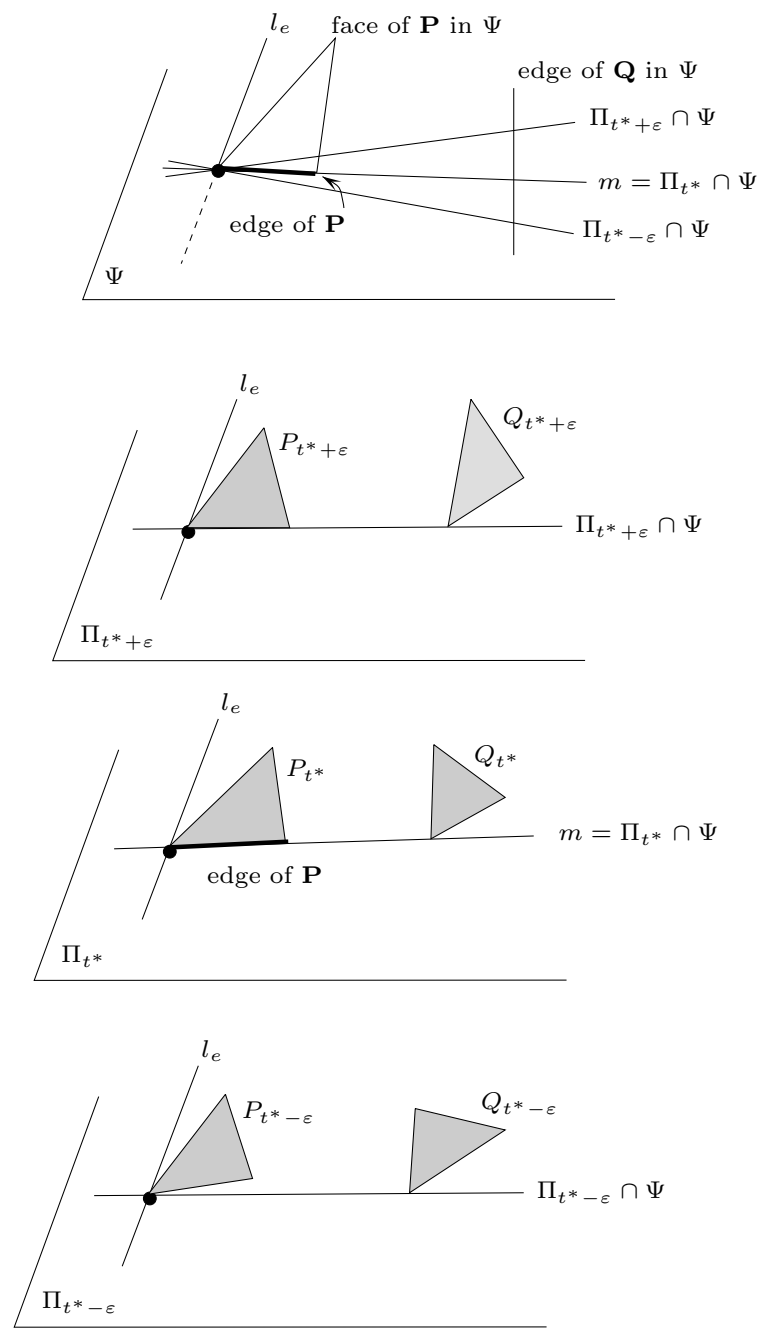

Figure 10: The set $u$ of supports of line $\Pi_{t^{*}-\varepsilon} \cap \Psi$ is a maximal $\left(P_{t}, Q_{t}\right)$-tuple for some but not all $t$ in any open neighborhood of $t^{*}$. There is a F-critical event $\left(t^{*}, m\right.$ ) (where $m=\Pi_{t^{*}} \cap \Psi$ ) such that $u$ is contained in the set of supports of $m$ because (i) $m$ lies in plane $\Psi \neq \Pi_{t^{*}}$ containing a face of $\mathbf{P}$ and (ii) $m$ is tangent to $\mathbf{P} \cap \Psi$ at some point not on $l_{e}$. Note that there is no $\mathbf{V}$-critical event $\left(t^{*}, v\right)$ such that $u$ contains $v$ or an edge with endpoint $v$, and there is no line $\ell \in \Pi_{t^{*}}$ such that (i) $\ell$ lies in a plane $\Upsilon \neq \Pi_{t^{*}}$ containing a face of $P$ and (ii) $\ell$ is tangent to $Q \cap \Upsilon$ at some point not on $l_{e}$; indeed $\ell$ is necessarily equal to $m$, if $\Upsilon=\Psi$ then $\ell$ is not tangent to $Q \cap \Upsilon$ and we can consider that the plane, distinct from $\Psi$, that contains $\ell$ and a face of $\mathbf{P}$ is equal to $\Pi_{t^{*}}$. 